NBER WORKING PAPER SERIES

\title{
INTEGRATED REGRESSORS AND TESTS OF \\ THE PERMANENT INCOME HYPOTHESIS
}

\author{
James H. Stock
}

Kenneth $D$. West

Working Paper No. 2359

\author{
NATIONAL BUREAU OF ECONOMIC RESEARCH \\ 1050 Massachusetts Avenue \\ Cambridge, MA 02138 \\ August 1987
}

We thank Ben Bernanke, Larry Christiano, Nils Gottfries, N. Gregory Mankiw, Charles Plosser, an anonymous referee and participants in a seminar at the University of Rochester for helpful comments and discussions, and the National Science Foundation for financial support. The research reported here is part of the NBER's research program in Economic Fluctuations'. Any opinions expressed are those of the authors and not those of the National Bureau of Economic Research. 
NBER Working Paper \#2359 August 1987

Integrated Regressors and Tests of the Permanent Income Hypothesis

\title{
ABSTRACT
}

\begin{abstract}
We use recent research on estimation and testing in the presence of unit roots to argue that Hall's (1978) $t$ and $F$ tests of whether consumption is predicted by lagged income, or by lags of consumption beyond the first, are asymptotically valid. A Monte Carlo experiment suggests that the asymptotic $t$ and $F$ distributions provide a good approximation to the actual finite sample distribution.
\end{abstract}

James H. Stock

Hoover Institution

Stanford, CA 94305
Kenneth D. West

Department of Economics

Princeton University

Princeton, NJ 08544 
1. Introduction

Are regression tests of the permanent income model of consumption valid when consumption and income have unit roots? Recent research on estimation and testing in the presence of unit roots has emphasized that standard procedures are often asymptotically valid even with stochastically growing regressors. In this paper, we use these developments to show that Hall's (1978) t-and F-tests of whether consumption is predicted by lagged income, or by additional lags of consumption beyond the first, are legitimate under some standard assumptions about the first differences of consumption and income.

This result might at first seem surprising, since in their study of Flavin's (1981) test of the permanent income model Mankiw and Shapiro (1985) presented dramatic evidence that "if income is indeed a random walk, then the standard testing procedure is greatly biased toward finding excess sensitivity" of consumption to current income ( $p$. 165). This evidence is of course consistent with the results of many other studies that show that standard procedures may be severely biased in the presence of unit roots (e.g., Fuller (1976), Nelson and Kang (1981)).

Standard procedures are not, however, always mis leading when the regressors have unit roots. Sims, Stock and Watson (1986) and West (1986) show that even with nonstationary regressors the usual testing procedures are asymptotically valid if a regression can be rewritten so that the coefficients of interest are on stationary, zero mean regressors. In particular, if consumption and income are difference stationary and cointegrated -- as is argued empirically in Campbell (1985) and Engle and Granger (1987), and as the permanent income model implies -- Hall's (1978) regression can be rewritten in just this way. The presence or absence of time trends is not relevant to this result (Sims, Stock 
and Watson (1986)). The essential distinction between Hall's (1978) and Mankiw and Shapiro's (1985) regressions are that Hall included a lag of consumption as a regressor, Mankiw and Shapiro (1985) did not. This seemingly minor discrepancy means that Hall's regression can be rewritten in the necessary fashion, Mankiw and Shapiro's cannot. This important example emphasizes the general proposition that integration and cointegration properties of the regressors matter for the distribution of test statistics. We make this argument precise in Section 2 .

Of course, the finite sample performance of these tests might differ substantially from that predicted by asymptotic theory. To investigate this possibility, in Section 3 we report the results of some Monte Carlo experiments based on a cointegrated model of consumption and income. The support for the asymptotic theory is dramatic: in a sample of size 100, $t$-and F-tests based on the usual $5 \%$ asymptotic critical values are found to reject in $4 \%$ to $6 \%$ of the trials.

We caution the reader that we are not suggesting that standard procedures are always valid in the presence of unit roots. The arguments concerning nonstandard distributions in Mankiw and Shapiro (1985), for example, are appropriate in many circumstances. Rather, we are explaining that standard testing procedures are appropriate in many other circumstances. Determining when the usual asymptotics apply requires a careful examination of the integration, trend and cointegration properties of the regressors.

2. The Mode1 and Tests

We adopt Flavin's (1981) interpretation of the permanent income model. Consumption $\left(C_{t}\right)$ equals permanent income, the annuity value of the sum of human 
wealth $\left(h_{t}\right)$ and nonhuman wealth $\left(w_{t}\right)$ :

(1)

$$
C_{t}=r w_{t}+r(1+r)^{-1} h_{t}
$$$$
h_{t}=E_{t} \sum_{j=0}^{\infty}(1+r)^{-j} y_{t+j},
$$$$
w_{t}=(1+r) w_{t-1}+y_{t-1}-C_{t-1} \text {. }
$$

In (1), $r$ is the real interest rate, $y_{t}$ is labor income, and $E_{t}$ denotes expectations conditional on the consumer's information set (assumed to be equivalent to linear projections). Thus consumption is proportional to the sum of human wealth -- the expected present value of future labor income -- and accumulated savings.

Flavin (1981) showed that (1) implies that the change in consumption equals the unpredictable change in the annuity value of labor income, i.e., that $\Delta C_{t}=$ $C_{t}-C_{t-1}=r\left(h_{t}-E_{t-1} h_{t}\right)=\varepsilon_{t}$. This leads to Ha11's (1978) famous conclusion that consumption follows a random walk: $C_{t}=C_{t-1}+\varepsilon_{t}$, where $E_{t-1} \varepsilon_{t}=0$.

Current savings can also be obtained from (1). Let $Y_{t}^{d}$ be disposable income, $Y_{t}^{d}=y_{t}+r w_{t}$. Substituting the expression for human wealth into the first expression in (1)

(2)

$$
\begin{aligned}
& Y_{t}^{d}-C_{t} \equiv y_{t}+r w_{t}-C_{t}=-r(1+r)^{-1} \sum_{j=0}^{\infty}(1+r)^{-j}\left(E_{t} y_{t+j}-y_{t}\right) \\
& =-\sum_{j=1}^{\infty} E_{t}(1+r)^{-j} \Delta y_{t+j} .
\end{aligned}
$$

Thus current savings is the negative of the expected present discounted value of changes in future labor income, as emphasized by Campbe11 (1985). This 
implies that $C_{t}$ and $Y_{t}^{d}$ are cointegrated in the sense of Granger (1983) and Engle and Granger (1987). Following Nelson and Plosser (1982) and Mankiw and Shapiro (1985), suppose first that $y_{t}$ has a unit root with possibly nonzero drift -- the case considered in our Monte Carlo experiments -- so that $\Delta y_{t}$ is stationary with possibly nonzero unconditional mean $\alpha$. Then (2) implies that $\mathrm{Y}_{t}^{\mathrm{d}}-\mathrm{C}_{t}=k+u_{t}$, where $k \equiv-r^{-1} \alpha$ and $u_{t}$ is stationary with mean zero and finite variance. That is, $\mathrm{Y}_{\mathrm{t}}^{\mathrm{d}}$ and $\mathrm{C}_{\mathrm{t}}$ individually have unit roots, but $\mathrm{Y}_{\mathrm{t}}^{\mathrm{d}}-\mathrm{C}_{\mathrm{t}}$ is stationary. ${ }^{1}$

Alternatively, suppose that $y_{t}$ is stationary with a nonzero mean; again, (2) implies $Y_{t}^{d}$ and $C_{t}$ are cointegrated. Although so far $Y_{t}^{d}$ and $C_{t}$ have been assumed to have zero drift, we generalize this to let $E \Delta Y_{t}^{d}=E \Delta C_{t}=\mu$, so that $Y_{t}^{d}-C_{t}$ is stationary.

Mankiw and Shapiro (1985) follow Flavin (1981) and consider testing the random walk prediction using detrended data. One of their tests is the t-test of $\pi=0$ in the regression

$$
\Delta C_{t}=\mu+\pi Y_{t-1}^{d}+\gamma t+\varepsilon_{t}
$$

Mankiw and Shapiro (1985) assume that income is a pure random walk with drift and that consumer uses only past income to forecast future income. Since in this framework $Y_{t}^{d}=C_{t}+k$, the test (3) is equivalent to regressing $C_{t}$ on $C_{t-1}$ and a time trend and testing whether the coefficient on lagged consumption is one, where $C_{t}$ is a random walk under the null. ${ }^{2}$ The theoretical and Monte Carlo results in Fuller (1976) and the Monte Carlo results in Mankiw and Shapiro (1985) show that there is a strong tendency towards incorrectly rejecting the nul1 hypothesis that $\pi=0$ in (3). Indeed, Mankiw and Shapiro (1985, Table 2) find that if the usual 5 percent critical value for a t-test is used in a sample 
of size 100, the nu11 is rejected 61 percent of the time. As illustrated in Banerjee et al. (1986), the poor finite sample performance is indicative of nonstandard asymptotic behavior of the $t$ statistic on $\pi$.

Neither Fuller's (1976) nor Mankiw and Shapiro's (1985) analysis, however, is relevant to the regressions of the form reported by Hall (1978),

$$
C_{t}=\mu+\beta C_{t-1}+\pi_{1} Y_{t-1}^{d}+\ldots+\pi_{p} Y_{t-p}^{d}+\varepsilon_{t},
$$

in which $\pi_{1}=\ldots=\pi_{\mathrm{p}}=0$, and, in genera1, $\mathrm{C}_{\mathrm{t}} \neq \mathrm{Y}_{\mathrm{t}}^{\mathrm{d}}$, under the nul1 hypothes is. Although either (3) or (4) can be used to test the random walk hypothesis, the statistical properties of the test statistics based on the two regressions are different indeed. The key statistical difference between the regressions (3) and (4) is that, even though income has a unit root, the coefficients on income in (4) can all be written as coefficients on mean zero stationary variables, whereas in (3) this is impossible. It is impossible for (3) since no linear combination of a time trend and a variable with a unit root is stationary. By contrast, the right hand side of (4) can be rearranged to yield

$$
\begin{gathered}
C_{t}=\left(\mu+\pi_{1} k+\ldots+\pi_{P} k\right)+\left(\beta+\pi_{1}+\ldots+\pi_{p}\right) C_{t-1}+\pi_{1}\left(Y_{t-1}^{d}-C_{t-1}-k\right)+\ldots \\
+\pi_{p}\left(Y_{t-p}^{d}-C_{t-1}-k\right)+\varepsilon_{t}
\end{gathered}
$$

or

$\left(5^{\prime}\right) C_{t}=m+\phi C_{t-1}+\theta_{1}\left(Y_{t-1}^{d}-C_{t-1}-k\right)+\ldots+\theta_{p}\left(Y_{t-p}^{d}-C_{t-1}-k\right)+\varepsilon_{t}$.

where $m=\mu+\pi_{1} k+\ldots+\pi_{p} k, \phi=\left(\beta+\pi_{1}+\ldots+\pi_{p}\right)$ and $\theta_{i}=\pi_{i}$. Since the OLS estimators 
of $\left\{\theta_{i}\right\}$ in $\left(5^{\prime}\right)$ are algebraically identical to the OLS estimators of $\left\{\pi_{i}\right\}$ in (4), the null hypothesis could be tested using either regression.

The fact that (4) can be rewritten as (5) means that some standard results hold (given, of course, some standard assumptions about $\Delta C_{t}$ and $\Delta Y_{t}^{d}$ ). When time is excluded as a regressor and consumption and income have nonzero drift, Theorem 1 of Sims, Stock and Watson (1986) and Theorem 3.1 of West (1986) imply that the OLS estimators of $\left\{\pi_{i}\right\}$ are jointly asymptotically normally distributed, converging at the rate $\mathrm{T}^{1 / 2}$. Theorem 2 of Sims, Stock and Watson (1986) and Theorem 3.4 of West (1986) imply that $t$ - or F-tests examining any or all of these estimated coefficients have the usual asymptotic distributions. The theorems in West (1986) show that these results hold even if $\varepsilon_{t}$ is conditionally heteroskedastic. ${ }^{3}$ The theorems in Sims, Stock and Watson (1986) show that these results hold even if a time trend is included on the right hand side of (4), and consumption and income have zero drift. The key condition is that it is possible to rewrite the equation so that the coefficients of interest are on mean zero stationary variables. ${ }^{4}$ The asymptotic normal distribution follows because the stationary and mean zero regressors $\left\{Y_{t-i}^{d}-C_{t-1}-x\right\}, i=1, \ldots, p$, are asymptotically uncorrelated with the other regressors in $\left(5^{\prime}\right)$. Thus $\left\{\hat{\theta}_{i}\right\}$ have the usual joint asymptotic normal distribution, and converge at the usual $\mathrm{T}^{1 / 2}$ rate. $^{5}$

It is useful to contrast the asymptotic distribution of the coefficients on lagged income with that of the coefficients on lagged stock prices, which Hall (1978) also used to test the unpredictability of consumption changes. Suppose that stock prices have a unit root, but that consumption and stock prices are not cointegrated. ${ }^{6}$ Then Ha11's (1978) F-tests of the predictability of consumption using $p$ lags of stock prices are not valid. However, since any $p-1$ 
of the coefficients on lagged stock prices can be rewritten as coefficients on mean zero stationary variables (lagged changes of the stock price minus their means), tests based on any p-1 of the p estimated coefficients on stock prices are valid. In particular, if $p>1$, the usual t-test on each of these coefficients, considered individually, provides a valid basis for testing the predictability of consumption.

We close this section by providing some intuition for the result that imposing the true restriction $\beta=1$ in (4) so sharply changes the asymptotic analysis. To be concrete, suppose that only one lag of income is included as a regressor $(p=1)$, and imagine for the moment that consumption and income are stationary. Then imposing a true coefficient on $\mathrm{C}_{t-1}$ (which would be less than one) would in general improve the efficiency of the estimate of the coefficient on $\mathrm{Y}_{t-1}^{\mathrm{d}}$; the greater the correlation between $\mathrm{Y}_{t-1}^{\mathrm{d}}$ and $\mathrm{C}_{t-1}$, the greater the gain from imposing the restriction. In the case at hand, $\mathrm{Y}_{t-1}^{\mathrm{d}}$ and $\mathrm{C}_{t-1}$ are cointegrated, so that they are perfectly correlated in the sense that the $R^{2}$ of a regression of consumption on income will converge to one. The efficiency gained asymptotically from imposing $\beta=1$ in (4) reflects this asymptotic perfect multicollinearity, with the coefficient on $\mathrm{Y}_{t-1}^{\mathrm{d}}$ in the constrained case converging to a "unit roots" distribution at the rate $\mathrm{T}$ rather than $\mathrm{T}^{1 / 2}$.

\section{Monte Carlo Results}

Might these asymptotic results provide a useful guide in practice? We investigated this question using Monte Carlo experiments of the mode 1 (1). Let changes in labor income consist of two independent white noise components, $e_{1 t}$ and $e_{2 t}$, so that the nondeterministic portion of the process for labor 
income evolves according to $y_{t}=y_{t-1}+e_{1 t}+e_{2 t}$. Suppose that the consumer knows the first component contemporaneously but knows the second component one period in advance, so that the consumer's information set is $\left\{e_{1 t-j}, e_{2 t-j+1} \mid j \geq 0\right\}$. It is straightforward to show that, under the model (1)

$$
\begin{aligned}
& \Delta Y_{t}^{d}=\mu+e_{1 t}+(1+r)^{-1} e_{2 t} \\
& \Delta C_{t}=\mu+e_{1 t}+(1+r)^{-1} e_{2 t+1}
\end{aligned}
$$

We have added the drift $\mu$ to account for a possible deterministic component of income and consumption growth. Thus $C_{t}$ and $Y_{t}^{d}$ have unit roots, but $\mathrm{C}_{\mathrm{t}}-\mathrm{Y}_{\mathrm{t}}^{\mathrm{d}}=(1+\mathrm{r})^{-1} \mathrm{e}_{2 t+1}$ is stationary.

The Monte Carlo simulations were performed using income and consumption series generated by (6) and (7) with $\left(\mathrm{e}_{1 \mathrm{t}}, \mathrm{e}_{2 t}\right) \sim \mathrm{N}\left(0, .5 \mathrm{I}_{2}\right)$ (where $\mathrm{I}_{2}$ denotes the 2x2 identity matrix) and with Mankiw and Shapiro's (1985) value for r ( $1.25 \%$ per period). Out of concern that the small sample distributions of the tests might be sensitive to the size of the drift (see Evans and Savin [1984]), the experiments were repeated for $\mu=0,0.3,0.6,1.0$, and 3.0 ; the $0.6 \mathrm{drift}$ matches the ratio of the mean change in quarterly real per capita disposable income to its standard deviation from 1959:1 to 1983:4, the value used by Mankiw and Shapiro (1985). Regressions of the form (4) with $p=1$ and $p=4$ were estimated, both excluding and including time as a regressor. The experiments were repeated 5000 times using $\mathrm{T}=50$ and $\mathrm{T}=100$ observations, respectively representing the final observations of 55 and 105 draws of consumption and income.

Table 1 contains the results of the t-tests based on the $p=1$ regressions. A comparison of the asymptotic and Monte Carlo quantiles when time is excluded 
from the regression (panel A) indicates that the t-statistic exhibits a slight negative shift when there is no drift; with a positive drift the distribution is shifted slightly to the right. The shift in the distribution is more marked when time is included as a regressor (e.g., with $\mathrm{T}=100$ the 5 per cent Monte Carlo percentile is -1.82 , while the asymptotic is -1.65). ${ }^{7}$ This shift diminishes as the sample size grows. Moreover, in all the cases in Table 1 , the two sided t-test rejections fall between 9.0 per cent and 11.3 per cent using the asymptotic 10 per cent critical value, supporting the use of the asymptotic theory.

The results for F-tests based on regressions with four lags of income are reported in Table 2. As in the case of the t-tests, the tendency to reject too often is slight, the worst case at the $10 \%$ level being a rejection of $13.6 \%$ using detrended data with $\mathrm{T}=50$. When the sample size is increased to 100 , this rejection fraction drops to 12.0. Final1y, differences in the size of the drift evident1y have little effect on the size the F-tests.

We close this section with some evidence on Mankiw and Shapiro's (1985) suggestion that spurious excess sensitivity to income in the regression (3) should be attributed to small sample consumption-income correlations induced by incorrectly detrending a random walk. It appears instead that the spurious sensitivity is mainly due to the shift in the asymptotic distribution of the estimator of $\hat{\pi}$ in (3) from a normal to Fuller's (1976) "unit roots" distribution. This may be seen in tests of $H_{0}: \pi_{1}=\ldots=\pi_{p}=0$ in the regression

$$
\Delta C_{t}=\mu+\pi_{1} Y_{t-1}^{d}+\ldots+\pi_{P} Y_{t-p}^{d}+\varepsilon_{t} \text {, }
$$


with $p=1$ or $p=4$. We generated 5000 Monte Carlo samples of size 105 (so $T=100$ ), and tested $\mathrm{H}_{0}$ using the usual asymptotic $t$ and $F$ critical values. The results are in Table 3. The effect of even a small drift is to shift the upper tail of the distribution of the t-statistic substantially to the right. However, for $\mu=0.6$ (approximately the standardized shift in U.S. disposable income), substantially more mass remains in the left tail than is predicted by the asymptotic normal approximation. Nonetheless, the percent rejections for the Fand two sided t-tests are satisfactory for all nonzero drifts considered; they are, of course, quite unsatisfactory for $\mu=0.8$

\section{Conclusions}

These results suggest three conclusions. First, while it is premature to conclude that the qualitative results in this paper are applicable to other regressions in which the right hand side variables are all integrated or cointegrated, ${ }^{9}$ the asymptotic distribution theory provides a remarkably good guide to the small sample properties of the test statistics in the simple model of consumption studied here.

Second, while "spuriously detrending" an integrated process can often lead to highly biased inference (Nelson and Kang [1981]), this is not the case for the test statistics studied here. The methodological lesson of this exercise is that it is important to examine, either theoretically or empirically, the joint integration properties of the regressors in question as a step towards obtaining asymptotically justifiable inferences.

Third, Hall's (1978) tests involving lags of income are asymptotically valid in the context of the model presented in this paper. This conclusion does not, however, mean that there is little evidence against the permanent income 
mode1. Such evidence can be found in a difference-stationary or cointegrated environment (e.g. Campbe11 [1985], Nelson [1987], Watson [1986], and West [1987]) and in the individual t-statistics for the stock price regressions in Ha11's (1978) original paper. 


\section{Footnotes}

1. More precisely, if $\Delta y_{t}$ has a finite spectral density, then the variance of the final expression in (3) will be finite, so that $\mathrm{Y}_{t}^{\mathrm{d}}-\mathrm{C}_{\mathrm{t}}$ will be stationary. Engle and Granger (1987) provide empirical evidence that real per capita nondurables consumption and income are cointegrated.

2. Actually, Mankiw and Shapiro (1985, p. 169) assume that consumption and income are detrended in initial regressions, and that $\Delta C_{t}$ is then regressed against $\mathrm{Y}_{t}^{\mathrm{d}}$. As they note in their footnote 4, however, this is numerically equivalent to estimating the trend simultaneously, as in our equation (3). 3. In such a case, it is appropriate to use the standard Hansen (1982) and Newey and West (1987) adjustment for conditional heteroskedasticity. West (1986) also shows that the standard formulas would apply if the disturbance $\varepsilon_{t}$ were autocorrelated, or if one were estimating (4) by two stage least squares or Hansen's (1982) two-step, two stage least squares (although neither of these is a relevant possibility, in the present example).

4. There is no unique way to rewrite $\left\{\pi_{i}\right\}$ as coefficients on mean zero, stationary variables. For example, an alternative approach would be to write $p-1$ of these as coefficients on $\left(\Delta Y_{t-i}^{d}-\mu\right), i=1, \ldots, p-1$. However, this is unimportant: the transformation is needed only to show the existence of such a rewriting, and tests based on all such transformed regression models will be asymptotically equivalent.

5. See Fuller (1976) and Sims (1978) for a similar conclusion in the estimation of a univariate $A R(p)$ process with a unit root. It may be useful to note that if consumption has no drift or a time trend is present, $\hat{\phi}$ converges to its limiting distribution at rate $\mathrm{T}$ and $\hat{\mu}$ and $\hat{\phi}$ are not asymptotically normal. Similarly, $\hat{\pi}$ in (3) converges to it limiting nonnormal distribution at rate $\mathrm{T}$. 
Conversely, if consumption has a drift and no time trend is present, $\hat{\phi}$ converges at rate $\mathrm{T}^{3 / 2}$ and $\hat{\mu}$ and $\hat{\phi}$ are asymptotically normal. Incidentally, the fact that $\dot{\phi}$ converges more rapidly than $\left\{\hat{\theta}_{i}\right\}$ means that in asymptotic hypothesis tests that involve both $\hat{\phi}$ and $\left\{\hat{\theta}_{i}\right\}, \hat{\phi}$ can be treated as known with certainty. This is a useful fact in some contexts (see Sims, Stock and Watson (1986)), though not in the present example.

6. For real, nondurables services and consumption, and the real $S$ and $P 500$ stock price index, per capita, 1950:1-1984:4, the nul1 hypothes is of no cointegration is not rejected at even the 20 per cent level by either the Engle and Granger (1987) augmented Dickey-Fuller test or the Stock and Watson (1986) $q_{f}^{\tau}(2,1)$ test.

7. When a time trend is included, we report results only for $\mu=0$, since the results using detrended data do not depend on the size of the drift.

8. The fact that $\hat{\pi}$ is less biased in Table $3 A$ than in Table $1 A$ is consistent with the more rapid convergence of $\hat{\pi}$ when the unit root in $C_{t}$ is imposed. See the discussion at the end of Section 2.

9. In particular, the asymptotic approximation might not work as well in a Monte Carlo experiment in which savings $C_{t}-Y_{t}^{d}$ were serially correlated. 


\section{$\underline{\text { References }}$}

Banerjee, Anindya, Juan J. Dolado, David F. Hendry, and Gregor W. Smith, 1986, "Exploring Equilibrium Relationships in Econometrics with Static Mode1s: Some Monte Carlo Evidence," Oxford Bulletin of Economics and Statistics 48, 253-277.

Campbe11, John Y., 1985, "Does Saving Anticipate Declining Labor Income? An Alternative Test of the Permanent Income Hypothesis," manuscript, Princeton University.

Engle, Robert F., and C.W.J. Granger, 1987, "Dynamic Model Specification with Equilibrium Constraints: Co-Integration and Error Correction," Econometrica 55, 251-276.

Evans, G.B.A., and N.E. Savin, 1984, "Testing for Unit Roots: II," Econometrica $52,1241-1260$.

Flavin, Marjorie A., 1981, "The Adjustment of Consumption to Changing

Expectations about Future Income," Journal of Political Economy 89, 974-1009.

Fuller, Wayne A., 1976, Introduction to Statistical Time Series (Wiley, New York).

Granger, C.W.F., 1983, "Co-integrated Variables and Error Correcting Mode1s," University of California at San Diego Discussion Paper 83-13.

Ha11, Robert E., 1978, "Stochastic Implications of the Life Cycle-Permanent Income Hypothesis: Theory and Evidence," Journal of Political Economy 86, 971-987.

Hansen, Lars Peter, 1982, "Large Sample Properties of Generalized Method of Moments Estimators," Econometrica 50, 1029-1054.

Mankiw, N. Gregory, and Matthew D. Shapiro, 1985, "Trends, Random Walks and Tests of the Permanent Income Hypothesis," Journal of Monetary Economics 16, 165-174.

Nelson, Charles R., 1987, "A Reappraisal of Recent Tests of the Permanent Income Hypothes is," Journal of Political Economy 95, 641-646.

Nelson, Charles R. and Heejon Kang, 1981, "Spurious Periodicity in

Inappropriately Detrended Time Series," Econometrica 49, 741-751.

Nelson, Charles R. and Charles I. Plosser, 1982, "Trends and Random Walks in Macroeconomic Time Series," Journal of Monetary Economics 10, 139-162.

Newey, Whitney K., and Kenneth D. West, 1987, "A Simple, Positive Semidefinite, Heteroskedasticity and Autocorrelation Consistent Covariance Matrix," Econometrica 55, 703-708.

Sims, Christopher A., "Least Squares Estimation of Autoregressions with Some Unit Roots," Center for Economic Research, University of Minnesota, Discussion paper no. 78-95 (March 1978). 
Sims, Christopher A., James H. Stock and Mark W. Watson, 1986, "Inference in Linear Time Series Models with Some Unit Roots," manuscript.

Stock, James H., and Mark W. Watson, "Testing for Common Trends," Harvard Institute for Economic Research Discussion Paper \#1222 (March 1986).

Watson, Mark W., 1986, "Univariate Detrending Methods with Stochastic Trends," Journal of Monetary Economics 18, 49-76.

West, Kenneth D., 1986, "Asymptotic Normality, when Regressors Have a Unit Root," Princeton University Woodrow Wilson School Discussion Paper No. 110.

West, Kenneth D., 1987, "The Insensitivity of Consumption to News about Income," National Bureau of Economics Working Paper No. 2252; forthcoming, Journal of
Monetary Economics. 
Table $1^{a}$

t-Tests Involving a Single Lag of Income

A. $C_{t}=\mu+B C_{t-1}+\pi Y_{t-1}^{d}+\varepsilon_{t}$

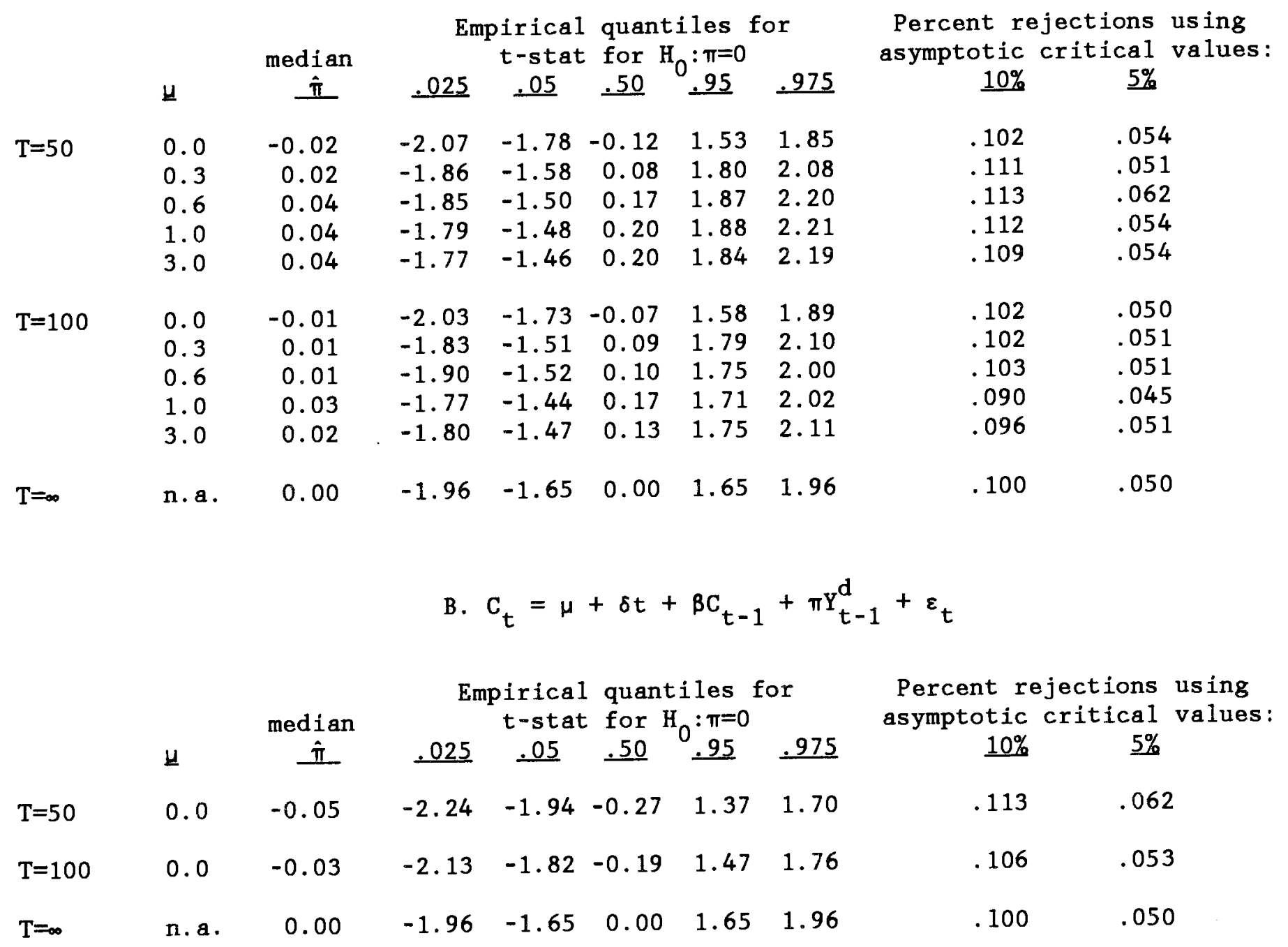

a. Distributions for $\mathrm{T}=50$ and $\mathrm{T}=100$ calculated from Monte Carlo simulation of 5000 draws on equations (6) and (7) $\left[\Delta Y_{t}^{d}=\mu+e_{1 t}+(1+r)^{-1} e_{2 t}, \Delta C_{t}=\mu+e_{1 t}+(1+r)^{-1} e_{2 t+1}\right]$, with $\left(e_{1 t}, e_{2 t}\right) \sim N\left(0, .5 I_{2}\right)$ and $r=.0125$. The $T=\infty$ lines report the asymptotic values. 
Table $2^{a}$

F-Tests Involving Four Lags of Income

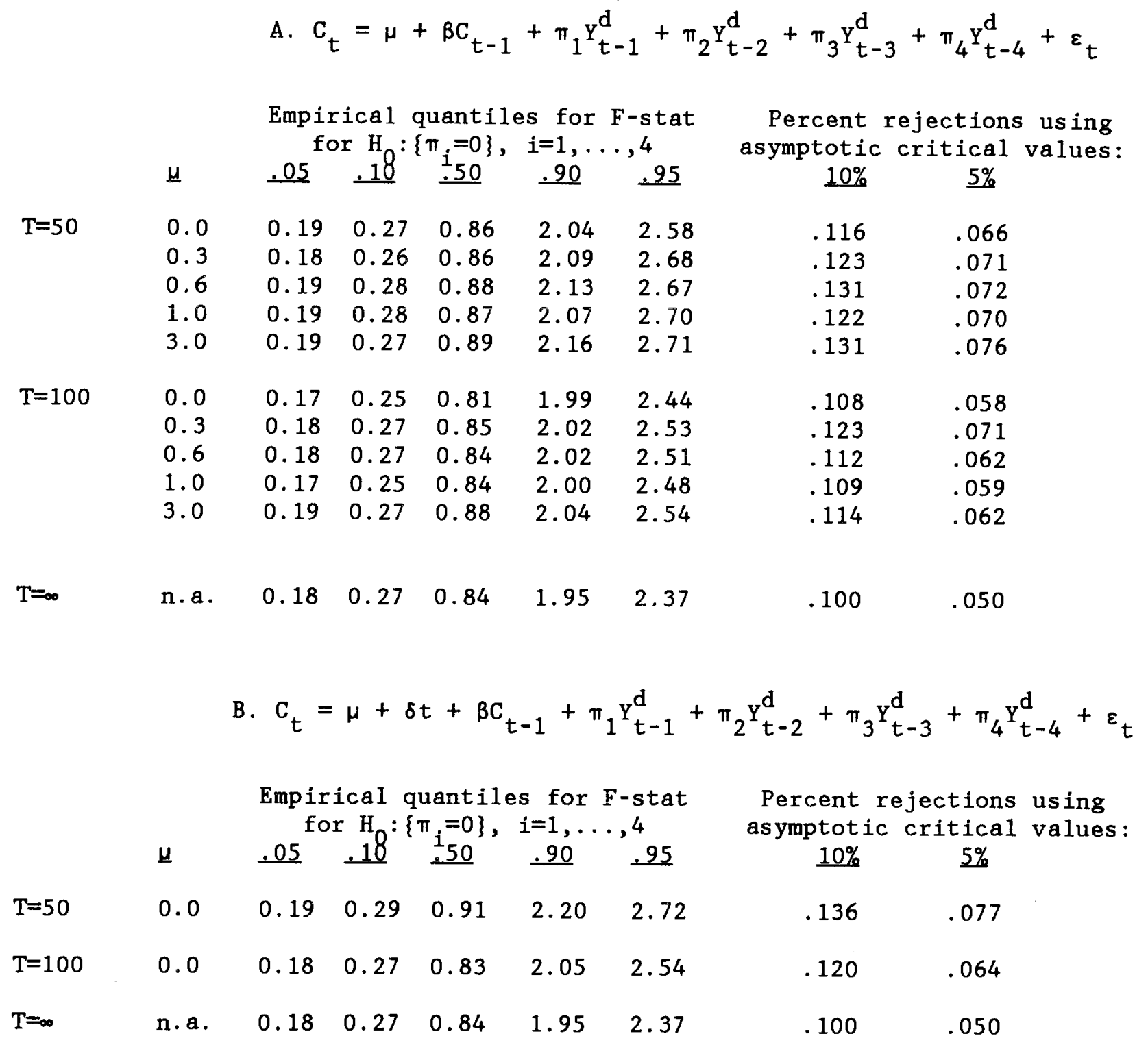

a. See note to Table 1 . 
Table $3^{a}$

Tests with $\Delta C_{t}$ as the Dependent Variable

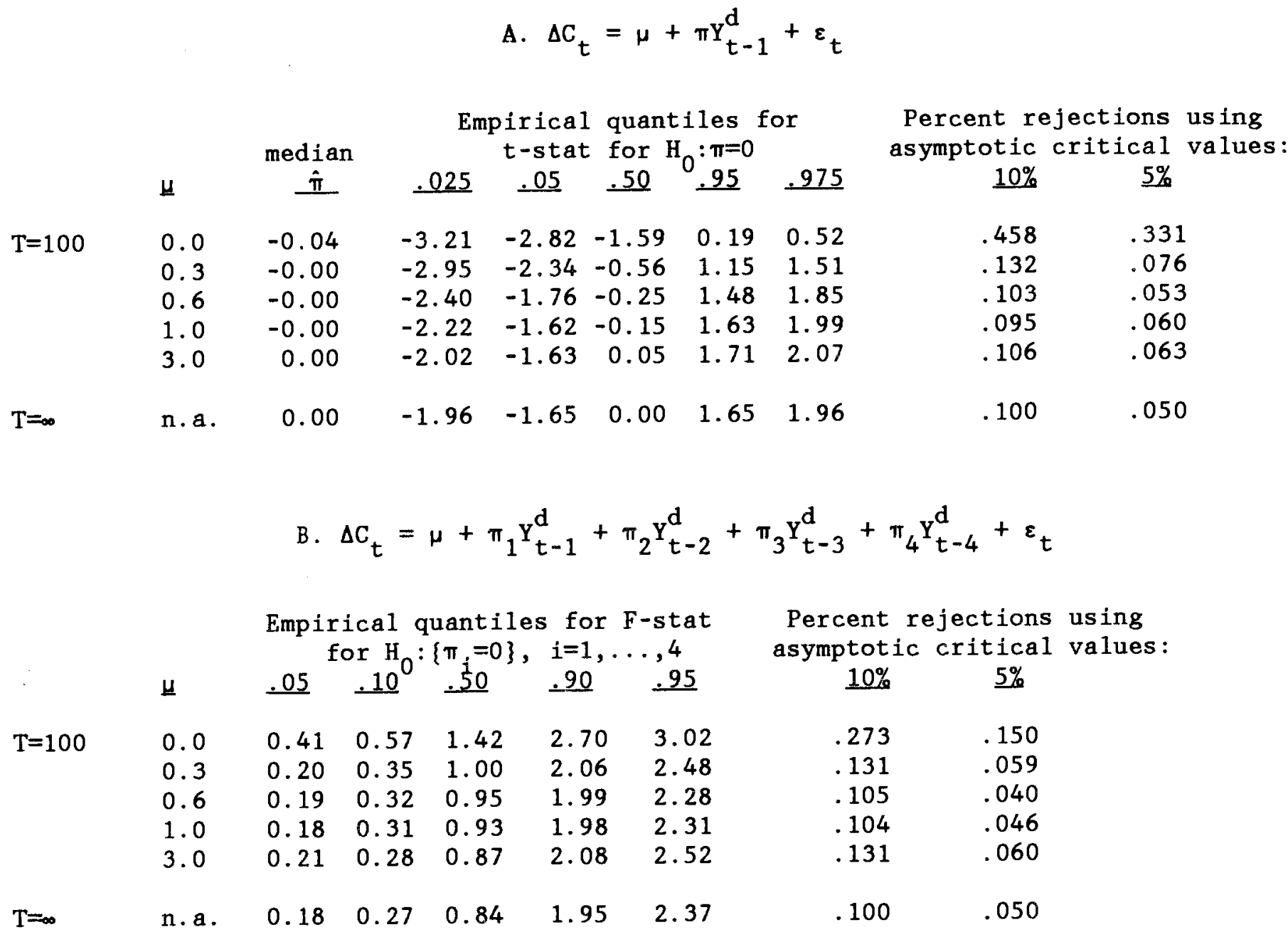

a. See note to Table 1. The entries for quantiles and percent rejections on the $T=\infty$ line do not apply when $\mu=0$, as explained in the text. 